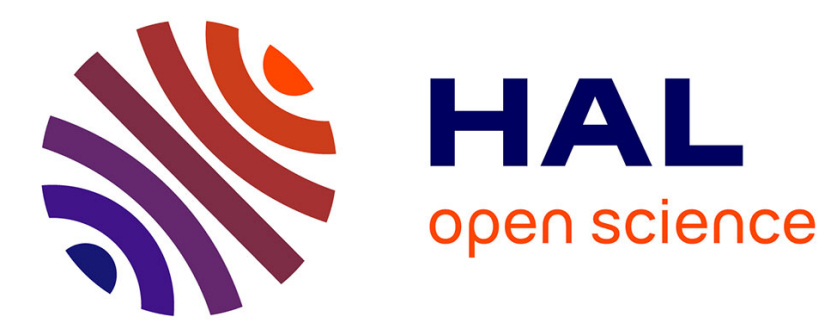

\title{
Recent developments in the study of rapid human movements with the kinematic theory: Applications to handwriting and signature synthesis
}

Réjean Plamondon, Christian O'reilly, Javier Galbally, Abdullah Almaksour, Éric Anquetil

\section{To cite this version:}

Réjean Plamondon, Christian O'reilly, Javier Galbally, Abdullah Almaksour, Éric Anquetil. Recent developments in the study of rapid human movements with the kinematic theory: Applications to handwriting and signature synthesis. Pattern Recognition Letters, 2014, 35, pp.225-235. 10.1016/j.patrec.2012.06.004 . hal-00801866

\section{HAL Id: hal-00801866 https://hal.science/hal-00801866}

Submitted on 4 Dec 2014

HAL is a multi-disciplinary open access archive for the deposit and dissemination of scientific research documents, whether they are published or not. The documents may come from teaching and research institutions in France or abroad, or from public or private research centers.
L'archive ouverte pluridisciplinaire HAL, est destinée au dépôt et à la diffusion de documents scientifiques de niveau recherche, publiés ou non, émanant des établissements d'enseignement et de recherche français ou étrangers, des laboratoires publics ou privés. 
Recent Developments in the Study of Rapid Human Movements with the Kinematic Theory: Applications to Handwriting and Signature Synthesis.

\title{
By Réjean Plamondon ${ }^{1}$, Christian O’Reilly ${ }^{1}$, Javier Galbally ${ }^{2}$, Abdullah
}

Almaksour $^{3}$, Éric Anquetil ${ }^{3}$,

\section{${ }^{1}$ Laboratoire Scribens, Département de Génie Électrique} École Polytechnique de Montréal, Canada rejean.plamondon@polymtl.ca

\author{
${ }^{2}$ ATVS - Biometric Recognition Group \\ Universidad Autonoma de Madrid, Spain
}

\section{${ }^{3}$ INSA de Rennes, France}

Abstract: Human movement modeling can be of great interest for the design of pattern recognition systems relying on the understanding of the fine motor control (such as online handwriting recognition or signature verification) as well as for the development of intelligent systems involving in a way or another the processing of human movements. In this paper, we briefly list the different models that have been proposed in order to characterize the handwriting process and focus on a representation involving a vectorial summation of lognormal functions: the Sigma-Lognormal model. Then, from a practical perspective, we describe a new stroke extraction algorithm suitable for the reverse engineering of handwriting signals. In the following section it is shown how the resulting representation can be used to study the writer and signer variability. We then report on two joint projects dealing with the automatic generation of synthetic specimens for the creation of large databases. The first application concerns the automatic generation of totally synthetic signature specimens for the training and evaluation of verification performances of automatic signature recognition systems. The second application deals 
28 with the synthesis of handwritten gestures for speeding up the learning process in

29 customizable on-line recognition systems to be integrated in electronic pen pads.

\section{Introduction}

31 Human movement modeling can be of great interest for the design of pattern recognition

32 systems relying on the understanding of the fine motor control, like on-line handwriting

33 recognition and signature verification, as well as in the development of intelligent

34 systems involving in some way the analysis of human movements. Among other things,

35 this general approach aims at elaborating a theoretical background for any handwriting

36 processing application as well as providing some basic knowledge that can be integrated

37 in the development of automatic systems.

38 So far, many models have been proposed to study human movement production in 39 general and handwriting in particular : models relying on neural networks (Bullock and

40 Grossberg, 1988; Schomaker, 1991; Gangadhar et al., 2007; Kalveram, 1998),

41 equilibrium point models (Feldman, 1966; Feldman and Latash, 2005; Bizzi et al., 1978;

42 1992), behavioral models (Schmidt, 1999; Thomassen et al., 1983; van Galen and

43 Teulings, 1983), coupled oscillator models (Hollerbach, 1981; Kelso, 1995; Zazone et al.,

44 2005), kinematic models (Plamondon, 1995; Plamondon and Djioua, 2006), and models

45 exploiting minimization principles (Wada and Kawato, 1995; Engelbrecht, 2001):

46 minimization of the acceleration (Neilson, 1993; Neilson and Neilson, 2005), of the

47 energy (Nelson, 1983), of the time (Tanaka et al., 2006; Enderle and Wolfe, 1987;

48 Hermes and LaSalle, 1969), of the jerk (Hogan, 1984; Flash and Hogan, 1985), of the

49 snap (Edelman and Flash, 1987), of the torque changes (Uno et al., 1989) and of the

50 sensory-motor noise (Harris and Wolpert, 1998). Finally, many models exploit the 
51 properties of various functions to reproduce human movements: exponentials

52 (Plamondon and Lamarche, 1986), second order systems (Denier van der Gon and

53 Thuring, 1965; Dooijes, 1983), gaussians (Leclerc et al., 1992), beta functions (Alimi,

54 2003), splines (Morasso et al., 1983) and trigonometrical functions (Maarse, 1987).

55 Among the models which provide analytical representations, the Kinematic Theory of 56 rapid human movements (Plamondon, 1995a, 1995b; Plamondon and Djioua, 2006) and

57 its Delta- and Sigma-lognormal models have been used to explain most of the basic

58 phenomena reported in classical studies on human motor control (Plamondon and Alimi,

59 1997) and to study several factors involved in the fine motricity (Djioua and Plamondon,

60 2008; O’Reilly and Plamondon, 2010; Woch et al., 2010). Apart from these fundamental

61 studies, the theory has been used, directly or indirectly, in many practical applications

62 like the design of a signature verification system (Plamondon, 1994), the development of

63 tools to help children learning handwriting (Djeziri, Guerfali, Plamondon, and Robert,

64 2002), as well as of biomedical set ups to detect fine motor control problems associated

65 with brain strokes (O’Reilly and Plamondon, 2011, 2012).

66 In this paper, we report on two new and original case studies dealing with the automatic

67 generation of synthetic handwritten specimens for the creation of large databases. The

68 first application addresses the automatic generation of totally synthetic signature

69 specimens which may be used for the training and evaluation of the verification

70 performances of automatic recognition systems as well as for the quality assessment of

71 specimens. The second application regards the synthesis of handwritten gesture for

72 speeding up the learning process in customizable on-line recognition systems to be

73 integrated in electronic pen pads. Sections 5 and 6 reports detailed results about these two 
74 genuine applications, which at the time of the ICFHR 2010 keynote address presented by

75 the first author, were the first trial of using the Kinematic Theory for the generation of

76 synthetic trajectories to be used in signature verification and gesture recognition

77 experiments.

78 To better understand these applications and estimate their potential interest, as well as

79 making the present paper self-consistent a brief survey of the Kinematic Theory is

80 presented in section 2, two algorithms used for sigma-lognormal parameter extraction are

81 outlined described in section 3 and the main results on previous studies of handwriting

82 variability are summarized in section 4. These sections present in a condensed and goal

83 oriented way, the main concepts and strategies that have been explored over the years and

84 that are necessary to understand the present applications, without coming back to these

85 complete and often more exhaustive studies.

87 2. The Kinematic Theory of Rapid Human Movement and its Sigma-

88 Lognormal Model

89 One key feature of the Kinematic Theory is that it relies on strong and robust

90 mathematical grounds. All the models that are used under this paradigm are based on the

91 lognormal function which has been proved to be the ideal curve for describing

92 asymptotically the impulse response of a neuromuscular network made up of a large

93 number of coupled subsystems controlling the velocity of a movement (Plamondon et al.,

94 2003). For simple reaching or pointing gestures, a target is specified and two of these

95 networks are needed to control a trajectory, an agonist network which is acting in the 
96 target direction and one antagonist, acting in the opposite direction. Overall, the speed

97 profile is then described by a Delta-lognormal equation, a weighted difference of two

98 lognormals (Plamondon, 1995a, 1995b). When more complex trajectories have to be

99 generated, like in handwriting or in signing, a sequence of targets has to be reached and,

100 globally, the trajectory of the pen tip can then be described by a vectorial summation of

101 lognormals, hereinafter called sigma-lognormal equations, which takes into account the 102 various changes of direction.

103 In this vectorial summation context, the production of a word or of a signature requires 104 the definition beforehand of an action plan that is made up of virtual targets, which are 105 linked in pairs with an arc of circle. This map of paired target points represents a 106 sequence of discontinuous strokes. This plan triggers a motor command generator that 107 produces a series of impulses activating the neuromuscular systems characterized by their 108 lognormal impulse response (Plamondon and Privitera, 1995). For each impulse, a 109 lognormal velocity profile is generated at the pen tip and the time superimposition of 110 these strokes results in a smooth and well controlled trajectory. According to this 111 representation, the original strokes are thus hidden in the signal.

112 Mathematically, the Sigma-Lognormal model considers the velocity of the pen tip, $\vec{v}(t)$, as 113 described by a vectorial summation of $N$ lognormal primitives:

$$
\vec{v}(t)=\sum_{i=1}^{N} \vec{v}_{i}(t)=\sum_{i=1}^{N} \vec{D}_{i}(t) \Lambda_{i}\left(t, t_{0 i}, \mu_{i}, \sigma_{i}^{2}\right) ; N \geq 2
$$

115 Each lognormal in this summation defines a stroke scaled in amplitude by a command 116 parameter $(D)$ and time-shifted by the time occurrence of this command $\left(t_{0}\right)$, any 117 individual stroke pattern being described by a lognormal time function: 


$$
\Lambda\left(t ; t_{0 i}, \mu_{i}, \sigma_{i}^{2}\right)=\frac{1}{\sigma_{i} \sqrt{2 \pi}\left(t-t_{0 i}\right)} \exp \left(\frac{-\left[\ln \left(t-t_{0 i}\right)-\mu_{i}\right]^{2}}{2 \sigma_{i}^{2}}\right)
$$

119 Each of these primitives is also assumed to occur around a pivot, and the evolution of the 120 angular position of the trajectory can be calculated using an error function (erf):

$$
\theta_{i}(t)=\theta_{s i}+\frac{\left(\theta_{e i}-\theta_{s i}\right)}{2}\left[1+\operatorname{erf}\left(\frac{\ln \left(t-t_{0 i}\right)-\mu_{i}}{\sigma_{i} \sqrt{2}}\right)\right]
$$

123 where $\theta_{s i}$ and $\theta_{e i}$ refer, respectively, to the starting and ending angular direction of each

124 stroke. In equations (2) and (3), $\mu_{i}$ and $\sigma_{i}$ represent correspondingly the logtime delay

125 and the logresponse time of the neuromuscular system as it reacts to the $i^{\text {th }}$ command

126 (Plamondon and Djioua, 2006).

127 Under these conditions, the synergy produced by the interaction and coupling of many of

128 these neuromuscular systems results in the sequential generation of a complex 129 handwriting sample or a signature pattern.

\section{Sigma-lognormal Parameter Extraction}

132 To use the Sigma-Lognormal model for analyzing human movements, it is necessary to 133 have an algorithm to solve the inverse problem in a fully automatic fashion, that is, to 134 extract the lognormal parameters that most adequately fit the experimental data. The 135 Sigma-Lognormal parameters are considered to be well estimated and fitted for statistical analysis if the SNR, defined in (4), is over $20 \mathrm{~dB}$. 


$$
S N R=10 \log \left(\frac{\int v_{x_{-} n}^{2}+v_{y_{n} n}^{2} d t}{\int\left(v_{x_{-} n}-v_{x_{-} \Sigma}\right)^{2}+\left(v_{y_{-} n}-v_{y_{-} \Sigma}\right)^{2} d t}\right)
$$

137 In this equation, $\left(v_{x_{-} n}, v_{y_{-} n}\right)$ are the experimental (numerical) velocity signals and

$138\left(v_{x_{-} \Sigma}, v_{y_{-} \Sigma}\right)$ are the velocity signals of the sigma-lognormal reconstruction.

139 In the last years, two complementary algorithms have been proposed to solve this 140 nonlinear regression problem, the Robust Xzero based algorithm and the prototype based

141 algorithm. The next subsections briefly overview the state-of-the-art regarding these 142 parameter extractors.

\section{3.1. The Robust Xzero based extractor}

145 The Robust Xzero (RX0) based extractor is a powerful algorithm that provides an 146 accurate set of sigma-lognormal parameters describing the end-effector trajectory (e.g. 147 the pen tip trajectory in handwriting studies) of arbitrarily complex motions without any 148 a priori knowledge regarding the nature of the movement. In the following text, an 149 outline of the algorithm is presented. A more comprehensive description can be found in 150 (O’Reilly and Plamondon, 2009; O’Reilly, 2012).

151 To implement this algorithm, sequences of five characteristic points $\left(t_{i_{-} n}, v_{t i_{-} n}\right)$ $152(i=1,2, . ., 5)$ must be located in the original speed signal $v_{t}$. Following a time occurrence 153 order, these points are: a local minimum, an inflexion point, a local maximum, an 154 inflexion point and a local minimum. The sigma-lognormal representation of these points 155 can be written as in equations (5-6) with the parameters $a_{i}$ defined in (7).

$$
t_{i_{-} \Sigma}=t_{0}+e^{\mu} e^{-a_{i}} \quad i \in\{1,2, \ldots, 5\}
$$




$$
\begin{gathered}
v_{t i \_}=\frac{D}{\sqrt{2 \pi}} e^{-\mu} \sigma^{-1} e^{\left(a_{i}-\frac{a_{i}^{2}}{2 \sigma^{2}}\right)} \quad i \in\{1,2, \ldots, 5\} \\
\left\{\begin{array}{l}
a_{1}=3 \sigma \\
a_{2}=1.5 \sigma^{2}+\sigma \sqrt{0.25 \sigma^{2}+1} \\
a_{3}=\sigma^{2} \\
a_{4}=1.5 \sigma^{2}-\sigma \sqrt{0.25 \sigma^{2}+1} \\
a_{5}=-3 \sigma
\end{array}\right.
\end{gathered}
$$

156 Nine estimators can be obtained for the values of the kinematic parameters $\left(t_{0 i}, D_{i}, \mu_{i}, \sigma_{i}\right)$ 157 corresponding to the nine different combinations $\left(t_{j_{-} n}, t_{k_{-} n}, v_{t l_{-} n}, v_{t m_{-} n}\right)$ (with $158 j, k, l, m \in\{2,3,4\}, l \neq m, j \neq k)$ in the equations (8)-(11).

$$
\begin{aligned}
& t_{0}=t_{j \_n}-e^{\mu} e^{-a_{j}} \\
& D=\sqrt{2 \pi} v_{t l_{-} \mathrm{n}} e^{\mu} \sigma e^{\left(\frac{a_{l}^{2}}{2 \sigma^{2}}-a_{I}\right)} \\
& \mu=\ln \left\{\frac{t_{j n}-t_{k, n}}{e^{-a_{j}}-e^{-a_{k}}}\right\} \\
& \sigma= \begin{cases}\sqrt{-2-2 \ln \left(\frac{v_{t I n}}{v_{t m_{-} n}}\right)-\frac{1}{2 \ln \left(\frac{v_{t l n}}{v_{t m_{-} n}}\right)}} & l \in\{2,4\}, m=3 \\
\sqrt{2 \sqrt{1+\ln ^{2}\left(\left(\frac{v_{t I n}}{v_{t m_{-} n}}\right)\right)}-2} & l=4, m=2\end{cases}
\end{aligned}
$$

159 The angular parameters $\left(\theta_{g}, \theta_{e}\right)$ associated with each estimation of the kinematic 160 parameter set $\left(t_{0 i}, D_{i}, \mu_{i}, \sigma_{i}\right)$ is obtained using (12)-(13), where $161 l_{3}=\int_{t_{0}}^{t_{s}} D \Lambda\left(t-t_{0} ; \mu, \sigma\right) d t=\frac{D}{2} \operatorname{erfc}\left(\frac{\sigma}{\sqrt{2}}\right)$ (with erfc being the complementary error 162 function defined as $\operatorname{erfc}(x)=1-\operatorname{erf}(x))$ and $\phi(t)$ is direction angle of the trajectory 163 with respect to time. 


$$
\begin{aligned}
& \theta_{s}=\phi\left(t_{3 \_n}\right)-\frac{d \phi\left(t_{3 \_n}\right)}{d t} l_{3} \\
& \theta_{e}=\phi\left(t_{3 \_n}\right)+\frac{d \phi\left(t_{3 \_n}\right)}{d t}\left(\mathrm{D}-l_{3}\right)
\end{aligned}
$$

164 A choice can be made between the nine estimations of the six sigma-lognormal 165 parameters by keeping the solution minimizing the error function (14).

$$
\int_{t_{1-n}}^{t_{s_{-} n}}\left(\left(v_{t}(t)-\widehat{D} \Lambda\left(t-f_{0} ; \hat{\mu}, \widehat{\sigma}\right)\right) \Lambda\left(t-f_{0} ; \hat{\mu}, \widehat{\sigma}\right)\right)^{2} d t
$$

166 It should be noticed that, before using the values $\left(t_{i_{-} n}, v_{t i_{-} n}\right)$ in the previous expressions, 167 some preprocessing should be applied to get proper estimations ${ }^{1}$. To extract the 168 parameters of a whole velocity signal, good results have been obtained by, first, 169 extracting sequentially (i.e. in increasing order of their time occurrence) the lognormal 170 components. For that matter, each lognormal is extracted and subtracted from the original 171 signal before proceeding to the next component. Then, a global non-linear optimization 172 process can be applied to improve the estimated values. If this approach results in an 173 unsatisfactory reconstruction SNR, more lognormal components can be extracted by 174 processing them by decreasing importance of their impact (assessed here by their relative 175 size) on the signal.

176 The latest improvements included in this extraction system have resulted in a significant 177 increase of the SNR fitting accuracy. For example, on a 683 signatures database

\footnotetext{
${ }^{1}$ The details of these preliminary computations are presented in (O'Reilly \& Plamondon, 2009; O’Reilly, 2012).
} 
178 comprising 124 subjects, an average increase of $7.9 \mathrm{~dB}$ has been obtained, passing from $17917.4 \mathrm{~dB}$ in (O'Reilly \& Plamondon, 2009) to $25.3 \mathrm{~dB}$. Fig. 1 gives an example of a 180 complex movement, in this case a signature, fitted using the proposed RX0 approach $181 \quad(\mathrm{SNR}=22.2 \mathrm{~dB})$.

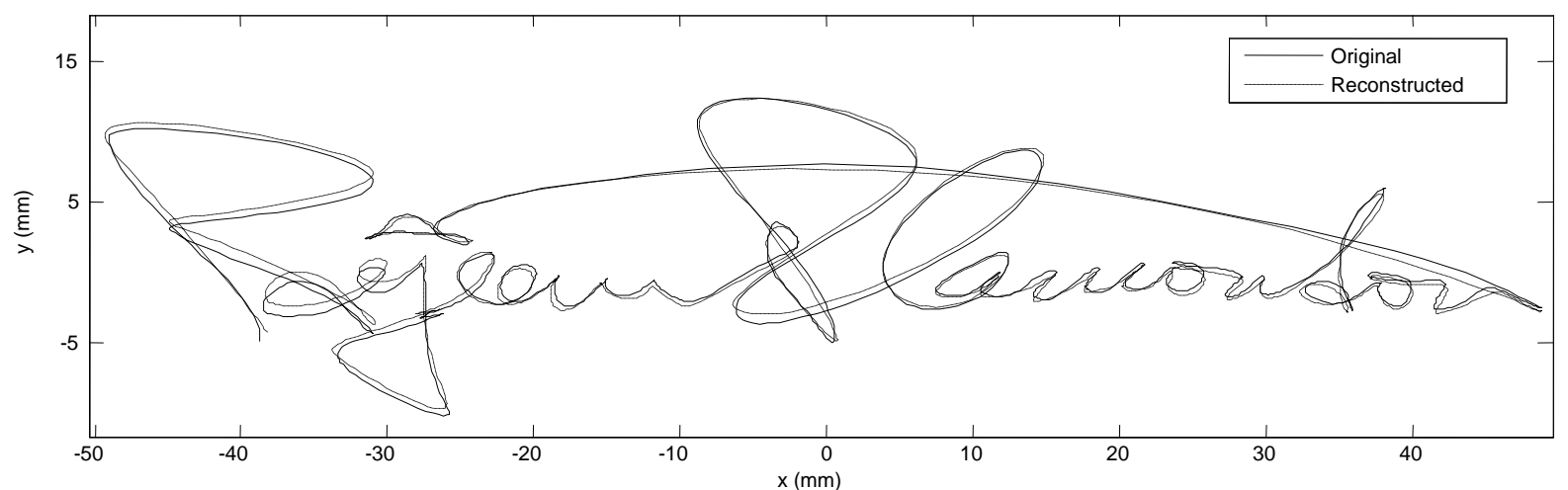

(a)

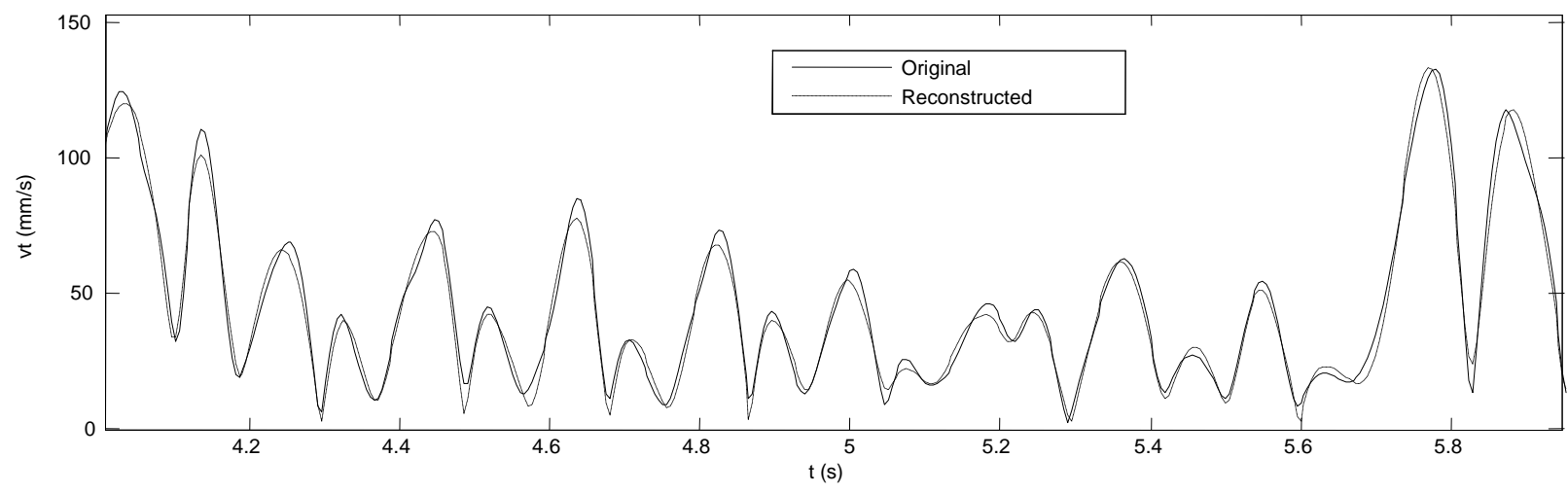

(b) 


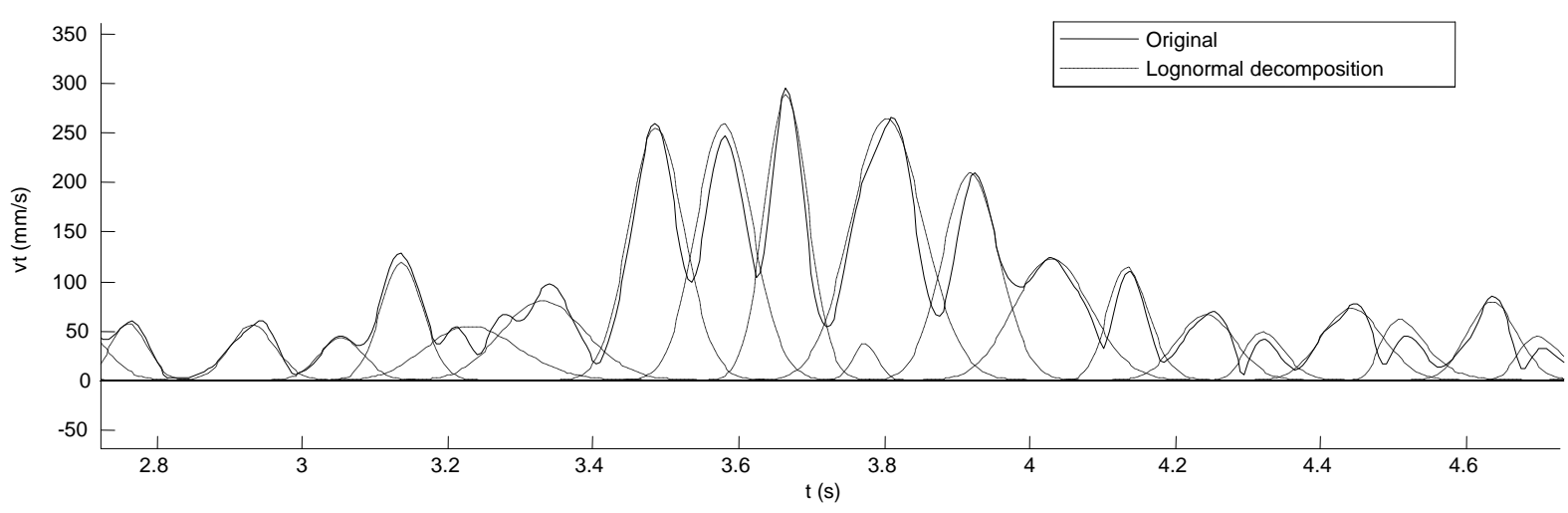

(c)

Fig. 1. Example of a signature reconstruction $(S N R=22.2 d B)$ following the proposed RX0 approach: (a) the trajectory, (b) the speed profile and (c) the lognormal decomposition of the speed profile. In (b) and (c), only a small part of the actual signals are shown to better allow the reader to appreciate the curve fitting and its lognormal decomposition.

\subsection{The prototype based extractor}

Although the system based on the Robust Xzero estimator gives very satisfying extraction results on complex movements, an alternative extraction strategy may be of great use under certain experimental scenarios. For example, this is the case of the analysis of stereotypical movements such as those often involved in psychophysical experiments. This type of experiments present some specific characteristics that can make an alternative extraction method better fitted for the task than RX0. First, a lot of a priori information on the nature of the movement is available which may be very helpful during the extraction process (the RX0 algorithm is not designed to take advantage of this knowledge). Second, in this kind of experimental framework, researchers may want to 
198 perform statistical testing of hypotheses on the value of local parameters. This may be 199 difficult with the solution obtained by an extractor such as the one based on RX0 200 because, in this case, there is no clear correspondence between the parameters extracted 201 from various movements.

202 These reasons supported the development of the prototype based extractor presented in 203 (O'Reilly and Plamondon, 2010). The advantages of such an extractor can be seen, for 204 example, in (O'Reilly and Plamondon, 2011) where it has been used to assess the 205 neuromuscular health of subjects on the basis of the sigma-lognormal parameters of their 206 movements.

207 This extractor applies a three step methodology: 1) synthesis of a sigma-lognormal 208 prototype of the stereotypical movements, 2) time scaling and offsetting of the prototype 209 to make it more closely correspond to the experimental movement, and 3) global 210 nonlinear optimization of the scaled and offset prototype to improve the fitting. These 211 three steps can be briefly described as follow:

212 - Step 1: Synthesis of the prototype. The initial prototype can be built from i) the 213 results of the RX0 extractor in order to find what is the expected sigma-lognormal 214 decomposition of the stereotypical movement or ii) from a sigma-lognormal 215 synthesizer such as SimScript (O’Reilly and Plamondon, 2007).

$216 \quad$ - Step 2: Movement scaling and offsetting. It is performed by finding the value of 217 the scaling $\left(C_{s}\right)$ and the offsetting $\left(t_{s}\right)$ factors that maximize the reconstruction SNR between the experimental data and the prototype signals modified in such a way that the original $\mu_{i}$ and $t_{0 i}$ parameters are changed for the scaled and shifted parameters $\mu_{i a}$ and $t_{\text {Oia }}$ according to (15)-(16). 


$$
\begin{aligned}
& t_{0 i a}=C_{s} t_{0 i}+t_{s} \\
& \mu_{i a}=\mu_{i}+\ln \left(C_{s}\right)
\end{aligned}
$$

221

222

223

224

225

226

227 Using this algorithm on a database of 1440 triangular movements performed by 120

228 subjects (see (O’Reilly \& Plamondon, 2011) for a more complete description of this

229 dataset), we obtained a mean SNR of $20.8 \mathrm{~dB}$ for the prototype based extractor compared

230 to a $22.1 \mathrm{~dB}$ for the RX0 based system. Although its SNR is slightly lower, the prototype

231 based extractor has the clear advantage of producing solutions with fixed number of

232 lognormals which enables the comparison among movements with any standard tool of 233 statistical analysis of variance.

\section{4. Automatic Generation of Trajectories: Variability Issues}

236 As we have seen, according to the Kinematic Theory, a complex movement results from

237 the superposition of a set of elementary movements (corresponding to single curved 238 strokes), localized both in time and space. So, the large variability observed in 239 handwriting patterns can be interpreted as caused both by the intrinsic variability of the 240 individual strokes and by fluctuations occurring in the time plan of the superimposition 241 process as controlled by the central nervous system. 
242 The local variability observed in handwriting and signing can thus come from various

243 sources. At the central level, a movement is represented by an action plan, a sequence of

244 virtual targets describing a piece-wise discontinuous trajectory. In parameter terms, this

245 plan is a timed sequence of arcs described by their length, directions and time of

246 activation. This discontinuous pattern, once instantiated, stimulates a set of

247 neuromuscular networks that react to each of these fundamental primitives with specific

248 time delays and response times.

249 In this context, at least three basic sources of variability can be identified:

250 1- a time variability associated with the temporal information contained in the 251 activation sequence of the different commands,

252 2- a spatial variability associated with the geometrical information contained in 253 commands themselves (the magnitude $D_{i}$ and direction $\left(\theta_{b i}\right.$ and $\left.\theta_{e i}\right)$ of each

254 stroke), and

2553 - a neuromuscular variability reflected in the timing properties $\mu_{i}, \sigma_{i}$ of the 256 neuromuscular networks reacting to these commands.

257 Those sources of variability have been investigated using a semi-automatic sigma258 lognormal parameter extraction methodology somewhat similar to the one described for 259 the prototype based extraction. Especially, we have shown that the Sigma-Lognormal 260 model, can explain the great variability of individual stroke trajectories and their 261 corresponding velocity profiles (Plamondon and Djioua 2005, 2006). We have also 262 applied this paradigm to study the possible sources of handwriting deformations caused 263 both by the disruptions in the motor control and the neuromuscular systems (Djioua and 264 Plamondon 2007, 2009). Particularly, we have shown that, without altering the rest of the 
265 factors involved in handwriting, the distortion of the shapes of a handwritten word is very 266 sensitive to slight changes of the time plan, represented by the sequence of command

267 time occurrences $\left\{t_{0 i}\right\}$. This stresses out the fact that, to write a readable word or to 268 generate a consistent signature, the production of strokes composing that word or 269 signature must be planned in advance in order to keep almost constant the timing of the 270 learned original plan. In contrast, the command parameters, that affect the direction $\left(\theta_{b i}\right.$ 271 and $\theta_{e i}$ ) and the amplitude $D_{i}$ of the strokes, seem to be less critical. And, finally, the

272 changes induced by the neuromuscular timing parameters $\mu_{i}, \sigma_{i}$ seem to have an even

273 smaller influence on the final variability of the trajectory.

274 Overall, these studies have shown the existence of a direct relationship between the 275 fluctuations of the sigma-lognormal parameters and the space and time warping of a pen 276 tip trajectory, which suggests the feasibility of using this model as a new tool for the 277 design of synthetic human like movements. This will be shown in the next two sections, 278 where the relative importance of the three sources of variability will be critical for the 279 successful design of large synthetic databases.

\section{5. Application 1: Automatic On-line Signature Database Generation}

282 One of the main obstacles that the biometric technology has found, and still finds, to 283 become one of the leading solutions in the security market, is the lack of large real 284 biometric databases which may serve as common benchmarks for the development of this 285 thriving technology. Two main reasons may explain such a scarcity of biometric data. On 286 the one hand, biometric database collection is not at all an easy job, involving a lot of 287 effort in terms of time and resources in order to reflect the variability present in biometric 
288 traits (both inter- and intra-class). This process includes a number of pre-acquisition and 289 post-acquisition demanding tasks such as the recruitment of donors, the supervision of 290 collected data, error correction or labeling (Flynn, 2008). On the other hand, biometric 291 traits are classified as personal data, and as such they are subdued to the different 292 personal data protection laws existing in each country, which makes the acquisition 293 (donor's consent), and later storage and distribution (licensing) of these data very 294 difficult.

295 Such a complex context has promoted over recent years the apparition of new algorithms 296 for the generation of synthetic biometric databases (Cappelli, 2003; Zuo, 2007). These 297 synthetically produced datasets are not affected by the acquisition and legal issues 298 mentioned before: $i$ ) first, once the appropriate generation method has been developed, 299 they are effortless to be produced, avoiding this way the arduous acquisition campaigns, 300 and ii) second, the synthetic samples which conform these databases cannot be 301 considered personal data as they have not been produced by a person, and so they may be 302 freely distributed in order to be used as common evaluation benchmarks. These desirable 303 characteristics make synthetic databases very powerful tools for the performance 304 assessment of biometric recognition systems, and have already been used in international 305 competitive evaluation campaigns (Cappelli, 2006).

306 In spite of presenting some very interesting features, the use of synthetic biometric 307 databases is not yet generalized as the production of realistic synthetic samples still 308 remains a challenging problem: modeling the information contained in a certain 309 biometric trait as well as the inter-class and intra-class variation found in real databases 310 (i.e., variation between samples of different subjects, and variation between samples of 
311 the same subject). Accepted solutions have been proposed for fingerprint (Cappelli,

312 2003) or iris (Zuo, 2007; Shah, 2006), but still no consistent method has been given for

313 the generation of synthetic handwritten signature databases.

314 As presented in previous sections, the Kinematic Theory of rapid human movements

315 provides a powerful theoretical framework which models in a precise and compact

316 manner the kinematic information involved in most of human writing processes,

317 including signature. Thus, the Kinematic Theory and its associated Sigma-Lognormal

318 model constitute a very high potential instrument for many different applications and

319 have been applied in the present work to the development of an algorithm for the

320 generation of fully synthetic on-line signatures.

\section{5.1. The generation method}

322 Two main parameters are involved in the design of real biometric databases and, hence,

323 should also be critical in the generation of synthetically produced datasets: $i$ ) number of 324 users comprised in the database, and ii) number of samples per user to be acquired. As 325 can be seen in Fig. 2, the generation method of synthetic signature databases proposed in 326 the present work is constituted of two different algorithms in order to produce: $i$ ) the first 327 sample of fully synthetic individuals (i.e., it allows to control the number of users in the 328 database), and ii) different samples derived from that original master signature (i.e., 329 permitting to fix the number of samples per user). 


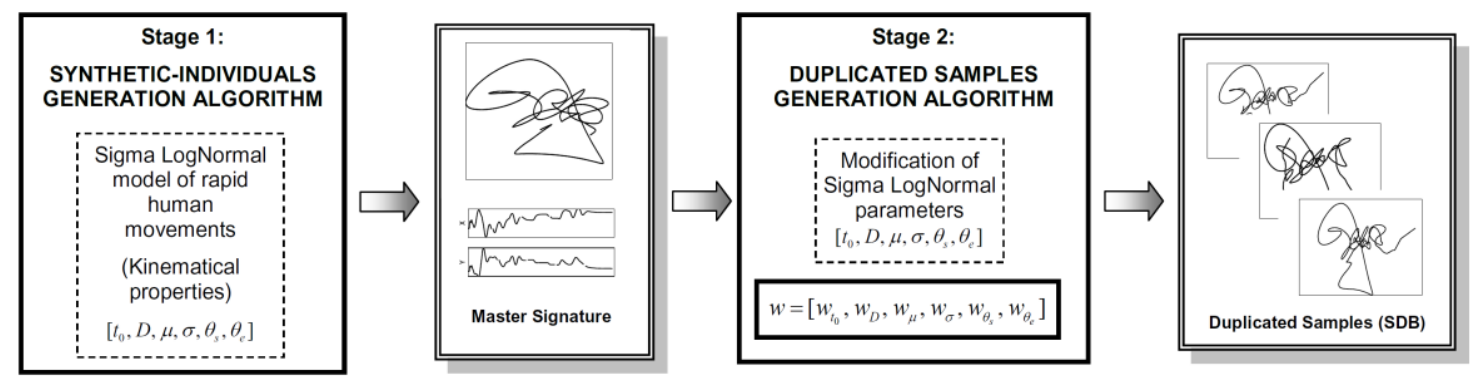

Fig. 2. General architecture of the algorithm proposed for the generation of synthetic signature databases based on the Kinematic Theory of rapid human movements.

\section{$330 \quad$ 5.1.1. Generating the master signature}

331 Although other signals such as the azimuth and elevation angles of the input pen or the

332 pressure applied during the signing process might be taken into account, in this work we

333 consider that an online signature is defined by two time sequences $[x[n] y[n]]$ specifying

334 respectively the $x$ and $y$ coordinates, at the time instants $n=1, \ldots, N$.

335 The objective of this initial stage of the generation algorithm is to produce samples from 336 different synthetic signers (i.e., this algorithm is responsible for controlling the number of 337 users in the database and for the inter-class variability present in the dataset).

338 In a first approach, a signature-like graphic is generated following the spectral approach 339 described in (Galbally, 2009). Although this first specimen has approximately the 340 appearance of a genuine signature, it does not possess many of the humanly produced 341 kinematic characteristics of real writing. Thus, in order to confer this preliminary master 342 signature with the velocity and acceleration properties of human strokes, it is processed 343 using the Sigma-Lognormal model in two consecutive stages, as shown in Fig. 3:

344 - Stage 1: Extraction of the sigma-lognormal parameters using the RX0 system. In this 345 phase, the velocity function of the initial synthetic master signature $\left(v_{I}\right)$ is decomposed 
346 in singular strokes and the sigma-lognormal parameters $\left(t_{0}, D, \mu, \sigma, \theta_{s}, \theta_{e}\right)$ which best

$347 \quad$ fit each of the individual strokes.

348 - Stage 2: Reconstruction of the velocity function of the definitive synthetic master 349 signature according to the previously computed parameters. The new coordinate 350 signals are then obtained from the reconstructed velocity function $\left(v_{D}\right)$ where we can 351 observe that certain abnormal artifacts such as the very high velocity peaks at the 352 starting and ending parts of the velocity profile have been corrected (see Fig. 3)

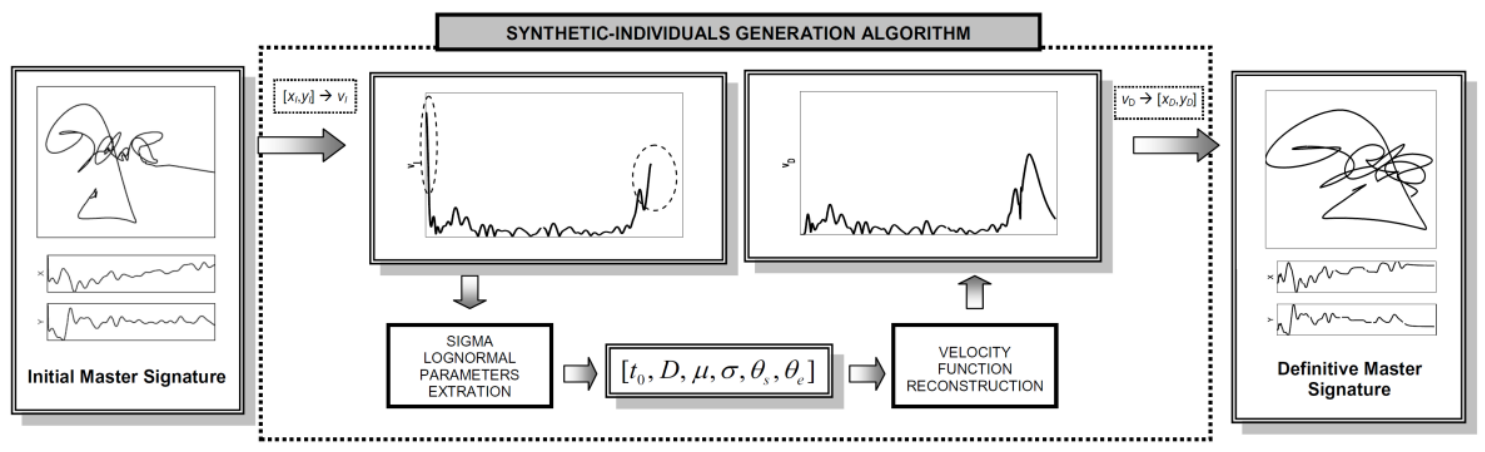

Fig. 3. General diagram of the generation process of a synthetic on-line master signature with the kinematic properties of a human-produced sample, based on the sigmalognormal parameters.

\section{$354 \quad$ 5.1.2. Generating the duplicated samples}

355 Once the time sequences $[x[\mathrm{n}] y[\mathrm{n}]]$ defining the master signature of a synthetic user have 356 been produced following the method described in Sect. 5.1.1, the next phase for the 357 automatic generation of synthetic on-line signature databases is the creation of duplicated 358 samples starting from that master sample (as is shown in Fig. 2). 
359 Therefore, the objective of this part of the proposed method is to produce different 360 samples of one same synthetic individual following the intra-variability found in real

361 signatures (i.e., existing variability among signatures produced by the same user).

362 For this purpose, the velocity function $v$ of the master signature is decomposed into single 363 strokes following the Sigma-Lognormal model where each stroke is defined by the set of 364 features $\boldsymbol{p}=\left[t_{0}, D, \mu, \sigma, \theta_{s}, \theta_{e}\right]$. The duplicated samples are then generated adding to each 365 of the single strokes a certain amount of noise which is modeled by a vector $\boldsymbol{w}=\left[w_{t 0}, w_{D}\right.$, $\left.366 w_{\mu}, w_{\sigma}, w_{\theta s}, w_{\theta e}\right]$ where $w_{t 0}$ is extracted from a uniform distribution $\left[-w_{t_{0}}^{\max } w_{t_{0}}^{\max }\right]$ which 367 is estimated according to the intra-user variability found in the development database 368 BiosecurID (Fierrez, 2010) (analogously for the rest of distortion elements comprised in 369 the vector $\boldsymbol{w}$ ). After the distortion stage, the new velocity function $v_{n}$ is computed, and in 370 a subsequent step the new coordinates $x_{n}$ and $y_{n}$ are recovered from that velocity 371 information (see Fig. 4).

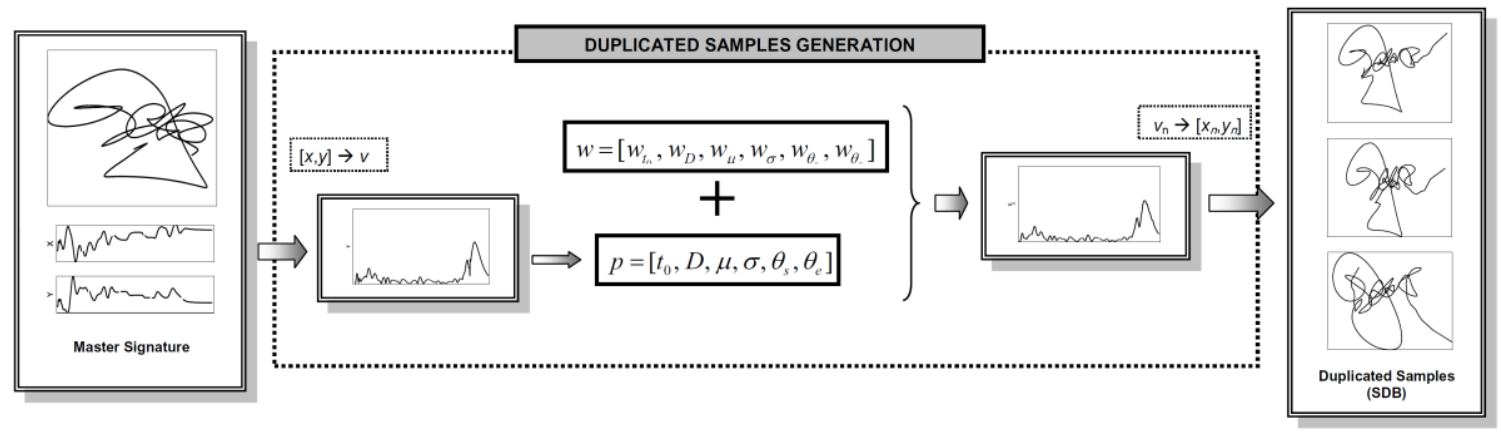

Fig. 4. General diagram of the generation process of duplicated samples starting from a fully synthetic specimen, based on the sigma-lognormal parameters.

373 In Fig. 5 some examples of synthetic signatures generated following the described 374 approach are shown, together with real samples extracted from the BiosecurID database 
375 (Fierrez, 2010) which was used as development set in order to compute the values of the different parameters involved in the generation method. As it can be observed from a

377 general visual comparison, the synthetic signatures, and especially their time functions,

378 present a very realistic appearance ${ }^{2}$ in terms of: 1 ) smoothness of the strokes; 2) growing
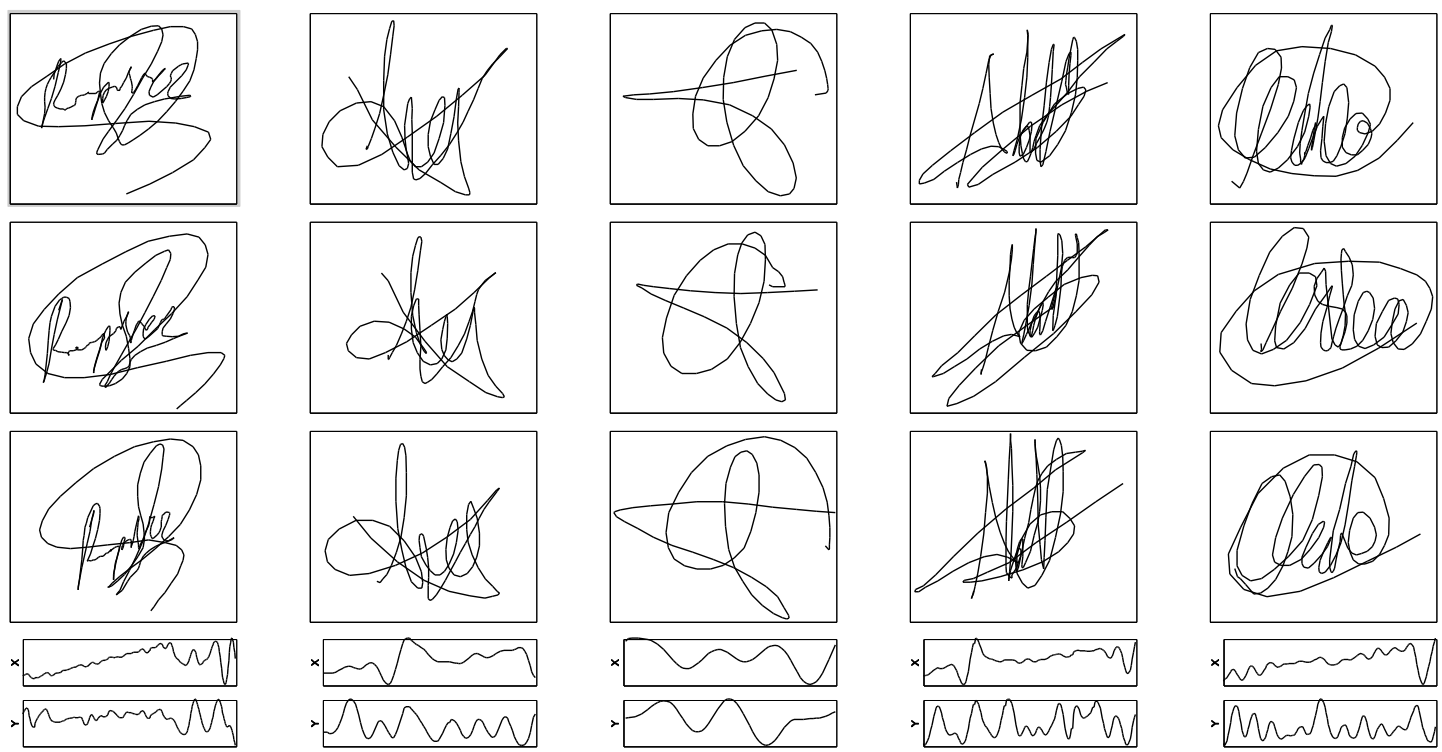

a) Real signatures extracted from the BiosecurID database.

\footnotetext{
${ }^{2}$ In this context, one concern that might be raised is dealing with the potential use of this methodology to fabricate forged signatures. This might be an issue except that for making realistic forgeries, the forger would need to have access to the complete on-line information about the target signature and if he does, there would be no need to use a complex methodology like the one described in this paper. The addition of some noise might be sufficient. However, the complete on-line information is generally not available in real life systems since it is generally not stored in the reference database.
} 

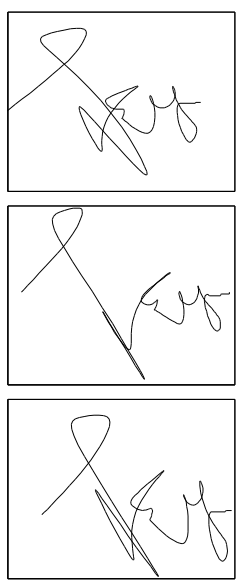

$\times \sqrt{n}$

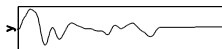

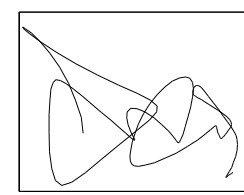
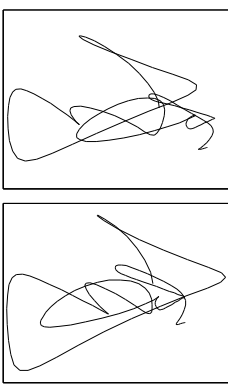

n r

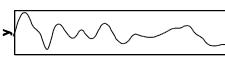

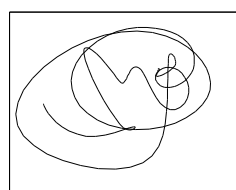
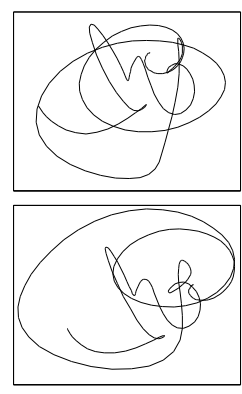

$\sim \sim$

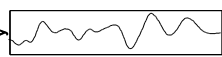

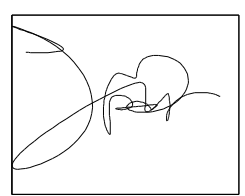
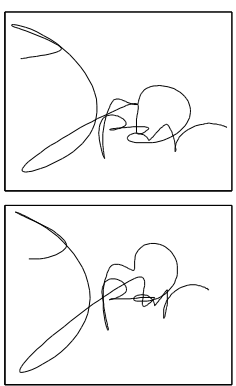

$\times$ r r

" varnar
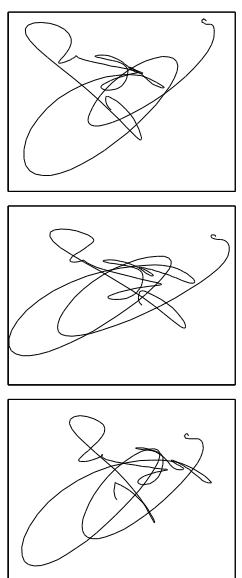

*hm N

N m N

b) Synthetic signatures produced with the proposed generation algorithm

Fig. 5. Examples of real (a) and synthetic (b) signatures extracted from BiosecurID and SDB. Three samples of 5 different real and synthetic signers are shown together with their time sequences $x[n]$ and $y[n]$ corresponding to the first sample.

379 tendency of the function $x$ (as it corresponds to left-to-right occidental signatures); 3)

380 large fluctuation at the end of the $x$ and $y$ signals in some of the signatures (corresponding 381 to some sort of round-like flourish); 4) degree of correlation between some of the most 382 relevant maxima and minima points in the $x$ and $y$ directions. Furthermore, even though it 383 is a model-based approach, some recognizable characters may be distinguished in the 384 synthetic samples.

\subsection{Validation experiments}

The experimental validation of the proposed generation method is aimed at determining if the performance of signature verification systems is similar when it is evaluated on real and synthetic databases. If the error rates presented by signature-based recognition applications is comparable in both scenarios (i.e., performance evaluation with real and 
391 synthetic signatures), it would mean that, from a computer-based perspective, the 392 synthetic signatures present a very similar behavior to that of real samples and that they 393 can be used to obtain a fair estimation of a system performance, avoiding this way the 394 different problems linked to real databases (i.e., high resource-consuming acquisition 395 campaigns and legal issues regarding their acquisition and distribution).

396 In order to achieve this objective, the performance of two signature verification systems, 397 using totally different feature sets and matchers, has been evaluated on the MCYT real 398 database (Ortega, 2003) and on a Synthetic DB (SDB) produced using the proposed 399 synthetic generation method.

400 The MCYT dataset has been selected as real test set since it has no overlap with the 401 BiosecurID database used as development set for the estimation of the generation method 402 parameters. This way we ensure to obtain totally unbiased results. The SDB has been 403 created with the same number of users (300) and samples per user (25) as MCYT in order 404 to permit the use of the same evaluation protocol for both scenarios.

405 The two on-line verification systems evaluated in the experiments are:

406 - System A: function based + HMM (Fierrez, 2007). This function-based verification 407 system applies a regional approach using a statistical model built using Hidden 408 Markov Models (HMMs) to a set of 10 time sequences selected applying the SFFS 409 feature selection algorithm (Pudil, 1994) to the total set of 34 functions defined in $410 \quad$ (Martinez-Diaz, 2009a).

411 - System B: function-based + DTW (Martinez-Diaz, 2009b). In this function-based 412 local approach, a subset of nine time functions (selected using the SFFS from the total 
34 feature set as in the case of system B) are directly matched using the elastic technique Dynamic Time Warping (DTW) (Kholmatov, 2005).

415 The performance results (Detection Error Trade-off, DET, curves) obtained for both 416 verification systems are shown in Fig. 6. We can observe that the curves of the two 417 systems present a very high degree of resemblance, both from a quantitative (EERs) and 418 qualitative (general behavior) point of view, for the case of real and synthetic signatures.

419 The results derived from this validation experiment confirm the great potential of the 420 Kinematic Theory of Rapid Human Movements applied to the generation of synthetic on421 line signature databases, and the suitability of such datasets to obtain reliable estimations 422 of the performance of signature verification systems.
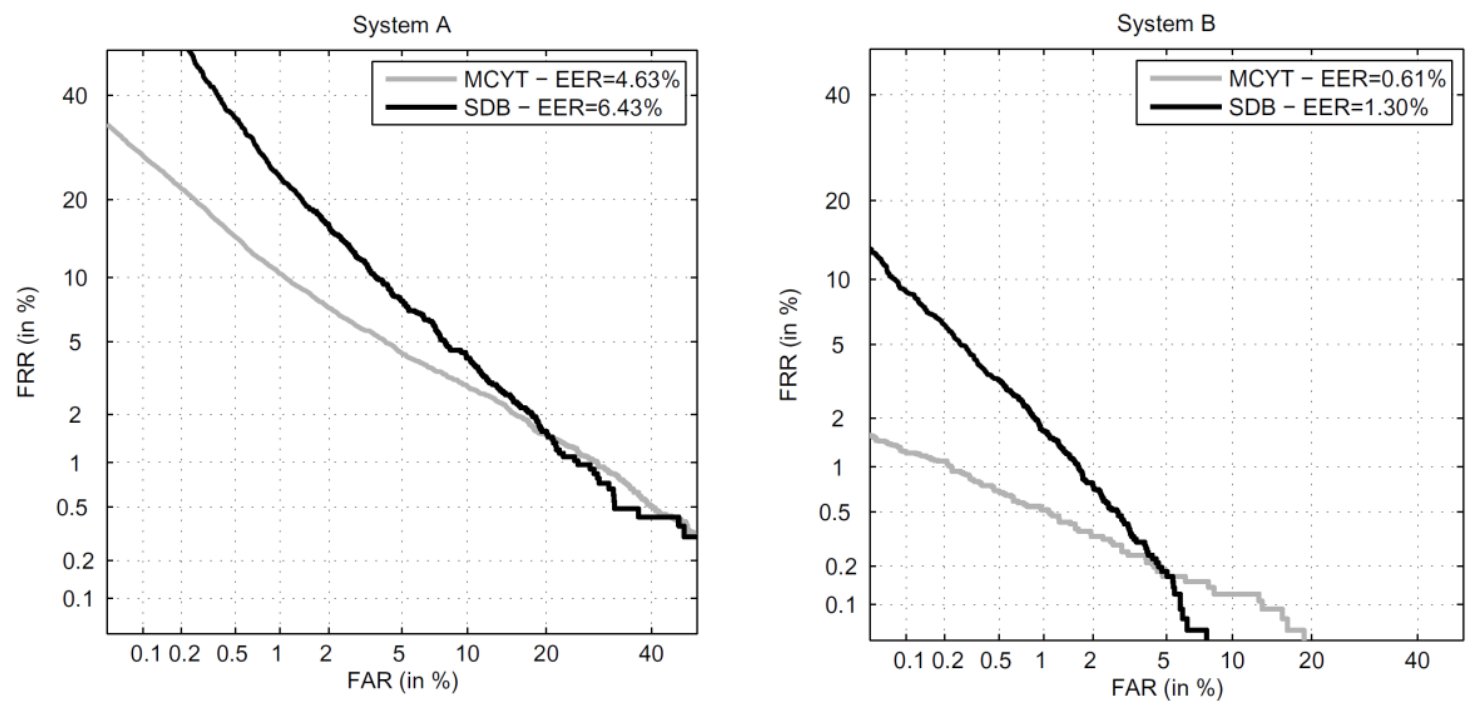

Fig. 6. Performance evaluation of systems A and B, on a real (MCYT, grey DET curve) and synthetic database (SDB, black DET curve). The EER is indicated in each plot.

\section{6. Application 2: Synthetic Gesture Generation for Evolving 425 Handwriting Classifiers}


426 Motivated by the increasing spread of many types of devices equipped with pen-based 427 interfaces, such as PDAs, e-book, Tablet PCs, Whiteboards, etc., more emphasis is placed

428 on the development of efficient recognition systems that can correctly interpret the 429 gestures sketched by the user and then translate them either into computerized text or into 430 some specific commands. Nowadays, the recognition systems in use are always pre431 trained on a fixed, predefined and a limited group of gestures, which usually contains the 432 Latin letters and a few specific gestures. These systems do not allow users to add gestures 433 in order to assign them to new commands or shortcuts, or to replace default gestures 434 mapped to existing commands. In order to meet this important functionality, the static 435 handwriting recognition systems that have been used so far must be replaced by novel 436 dynamic ones where the knowledge base can constantly evolve during the use of the 437 system. The evolving nature comes from the fact that the system must be able to integrate 438 at any moment a new class (gesture in our context), and must also continue its adaptation 439 to the existing classes using the new available data. Although the dynamic nature of 440 evolving classifiers offers many important advantages, the operation of these systems 441 suffers from the lack of learning data. The training process is done directly by the final 442 user in an online and interactive manner, so that the quantity of teaching samples is 443 limited because it is impractical to ask the user to enter a large number of samples in 444 order to obtain a functional classifier. Therefore, the main challenge in the conception of 445 incremental learning algorithms of evolving classification systems consists in reaching 446 high recognition performance as fast as possible; i.e. with the minimum number of 447 samples. Besides the beginning of the incremental learning from scratch, the problem of 448 lack of data samples appears again during the adaptation process when new classes are 
449 added to the classifier. The evolving system is supposed to be able to learn these new

450 classes without forgetting the old ones. However, it is difficult to completely avoid

451 perturbations on the global performance of the classifier when adding new classes and the

452 efforts must be focused on reducing as much as possible these perturbations.

453 In addition to the efforts of improving the classification systems and the training

454 algorithms, the incremental learning process can be further accelerated and enhanced by

455 generating artificial data based on some knowledge related to the application domain. For

456 handwritten gesture recognition problems, this idea can be implemented by generating

457 synthetic gestures from the available real ones after applying on them some deformations

458 in a realistic and significant manner. Thus, when a new class of gestures is introduced to

459 the system with few samples provided by the user, many artificial samples can be

460 generated. Geometric distortions are usually applied on real handwritten symbols in order

461 to generate synthetic ones (Mitoma, 2005; Wang, 2005; Lin, 2007; Mouchère, 2007).

462 These deformations can be either based on class-dependent models of gesture variability

463 and require a learning phase, or on class-independent general strategies without specific

464 deformation models.

465 In this work, we incorporate a handwriting generation technique using class-independent

466 lognormal-based deformations in the incremental learning of evolving handwritten

467 gesture classifiers. Thanks to the RX0 based extractor, the $\Sigma \Lambda$ parameter extraction and

468 the data generation is performed automatically as a part of the adaptation process. Motion

469 pattern variability rooted in the motor representation space of the handwritten gestures is

470 regarded to be more realistic than geometric distortions and thus more valuable in the

471 training process. In addition to the great advantage of integrating the lognormal-based 
472 handwriting generation technique in our evolving handwriting classifier, an objective and

473 numerical evaluation of the quality of generated data is provided for the first time, to the

474 best of our knowledge. The generated handwritten samples are considered realistic as

475 much as they help the classifier to predict future real samples from the same class of 476 gestures. The capacity of prediction is translated by the improvement of recognition 477 performance of the evolving classifier.

\section{6.1. Acceleration of the learning process using synthetic data}

480 As aforementioned, we believe that distortions obtained by applying some variations on 481 lognormal parameters are more realistic that those obtained using direct geometrical 482 distortions. The idea is to extract the sigma-lognormal profiles of a real handwritten 483 gesture provided by the user. Then, we apply some variation on the extracted parameters 484 within some specific ranges, and we regenerate artificial gestures using the modified 485 profiles. The resemblance between the synthetic and the real gestures is controlled by the 486 variation intervals. Thus, a suitable setting of these intervals is required in order to avoid 487 over-deformed gestures. We show in Fig. 7 some examples of the artificial gestures that 488 can be generated by applying modifications on the sigma-lognormal parameters. We can 489 note that the real gestures can be almost predicted from the synthetic ones. 


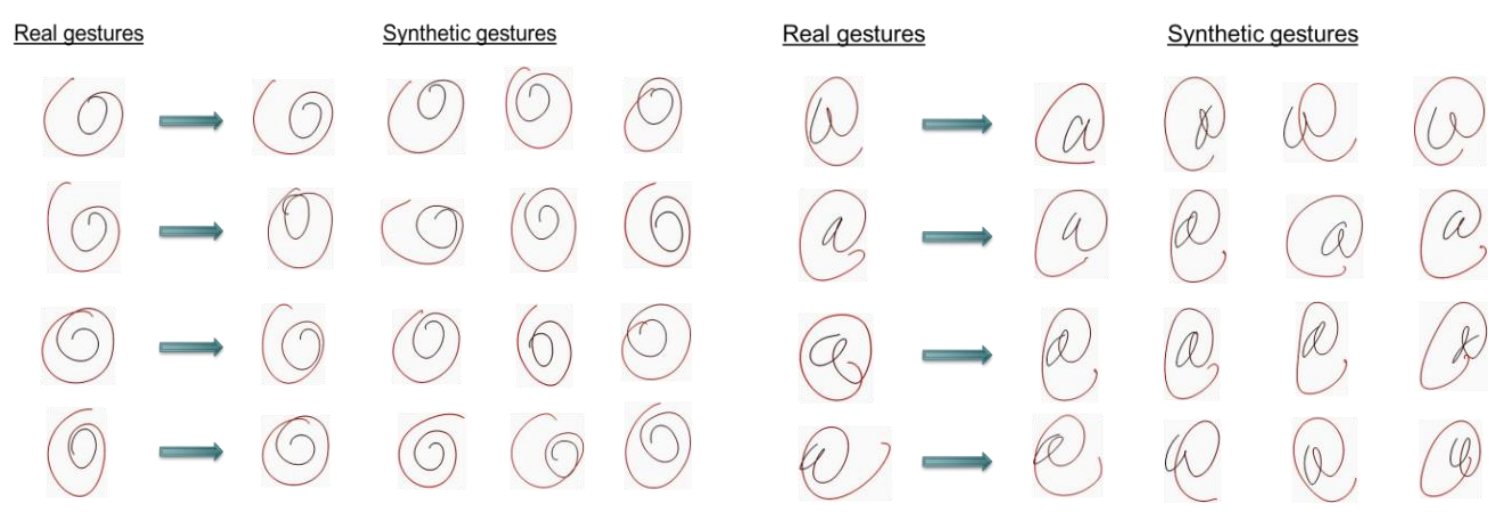

(a)

(b)

Fig. 7. Some examples of generated gestures using sigma-lognormal based deformations. 490

491 In the context of incremental learning of evolving systems, one can overcome the 492 problem of lack of samples at the beginning of the inclusion of a new class by generating, 493 in an adequate manner, a number of synthetic samples. For an evolving handwriting 494 classifier, the abovementioned sigma-lognormal based technique for synthetic gesture 495 generation can be incorporated into the incremental training process. The handwriting 496 generation is automatically performed transparently, with no user intervention. Fig. 8 497 shows the different steps of the generation method. Frist, the sigma-lognormal parameters 498 of each incoming sample are first extracted. These parameters are then modified and a 499 number of synthetic samples are generated. The original sample and the synthetic ones 500 are then introduced in the incremental learning algorithm.

501 Using SimScript (O’Reilly and Plamondon, 2007), a visualization interface developed by 502 the Scribens laboratory that allows an interactive modification of the sigma-lognormal 503 parameters of a given handwritten gesture, we have experimentally studied the valid 504 variation intervals of the six parameters within which the generated gesture is generally 505 considered similar (from a human viewpoint) to the original one. 


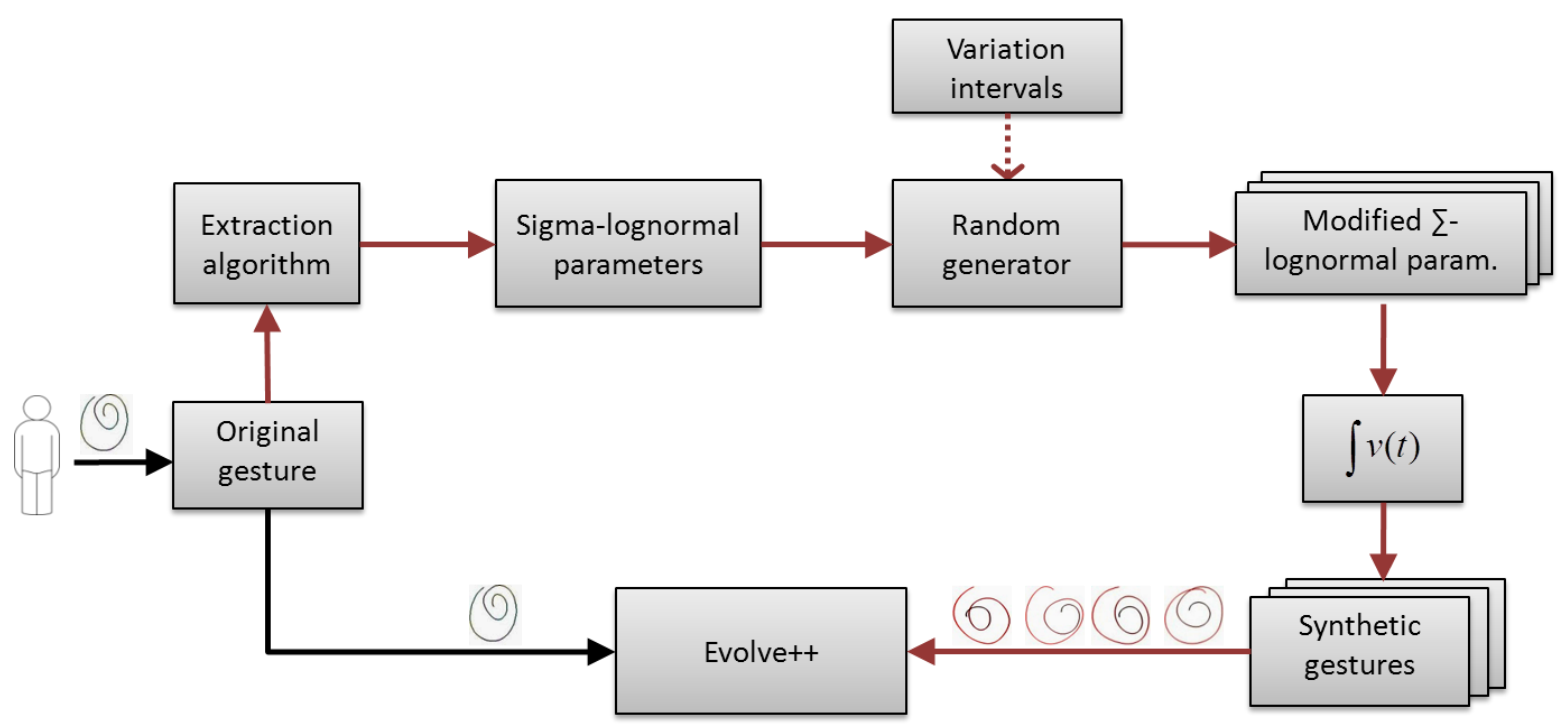

Fig. 8. Incorporating lognormal-based data generation into the learning stage of an evolving handwriting classifier.

\section{6.2. Experimental results}

508 Experiments have been performed on a dataset of on-line handwritten gestures. It was 509 composed of 11 different gestures drawn by 7 writers on a Tablet PC. Each writer has 510 drawn 100 samples of each gesture, i.e. 1,100 gestures in each writer specific dataset.

511 Each gesture was described by a set of 10 features. The presented results are the average 512 of 7 different tests for the 7 writers. In order to avoid the data order inducing a bias into 513 the outcome, we repeated 40 times the experiment for each writer with different random 514 data orders and only the averages are reported. We have used about $40 \%$ of the dataset 515 for the incremental training and the rest is used to estimate the evolution of the 516 performance during the learning process. We generated 10 synthetic samples (gestures)

517 for each real sample. The evolving classifier used in these experiments is based on a first- 
518 order Takagi-Sugeno (TS) fuzzy inference system, and taught with our original 519 incremental learning approach "Evolve++” (Almaksour, 2011).

520 We compared the lognormal-based handwriting generation method to the geometric 521 distortions explained in (Mouchère, 2007). Therefore, three performance curves are 522 presented in the figures:

523 I. Evolve++: our evolving classification approach with Evolve++ algorithm presented 524 in (Almaksour, 2011). Only real samples are considered (no synthetic data);

525 II. Evolve++\&Geo: synthetic samples are generated by applying geometric distortions 526 and used along with real ones to train Evolve++ system;

527 III. Evolve++\&Sigma: synthetic samples are generated using the lognormal-based $528 \quad$ method.

529 The results are presented for two different experimental scenarios: the 11 gestures were 530 introduced together in the first case, while the gestures were progressively introduced in 531 the second one. We measure in the former the impact of the synthetic samples at the 532 beginning of the learning process from scratch, while the latter scenario aims at showing 533 the impact of these synthetic samples when introducing new gestures. The results of the 534 first set-up are presented in Fig.9.a and those of the second in Fig.9.b. 


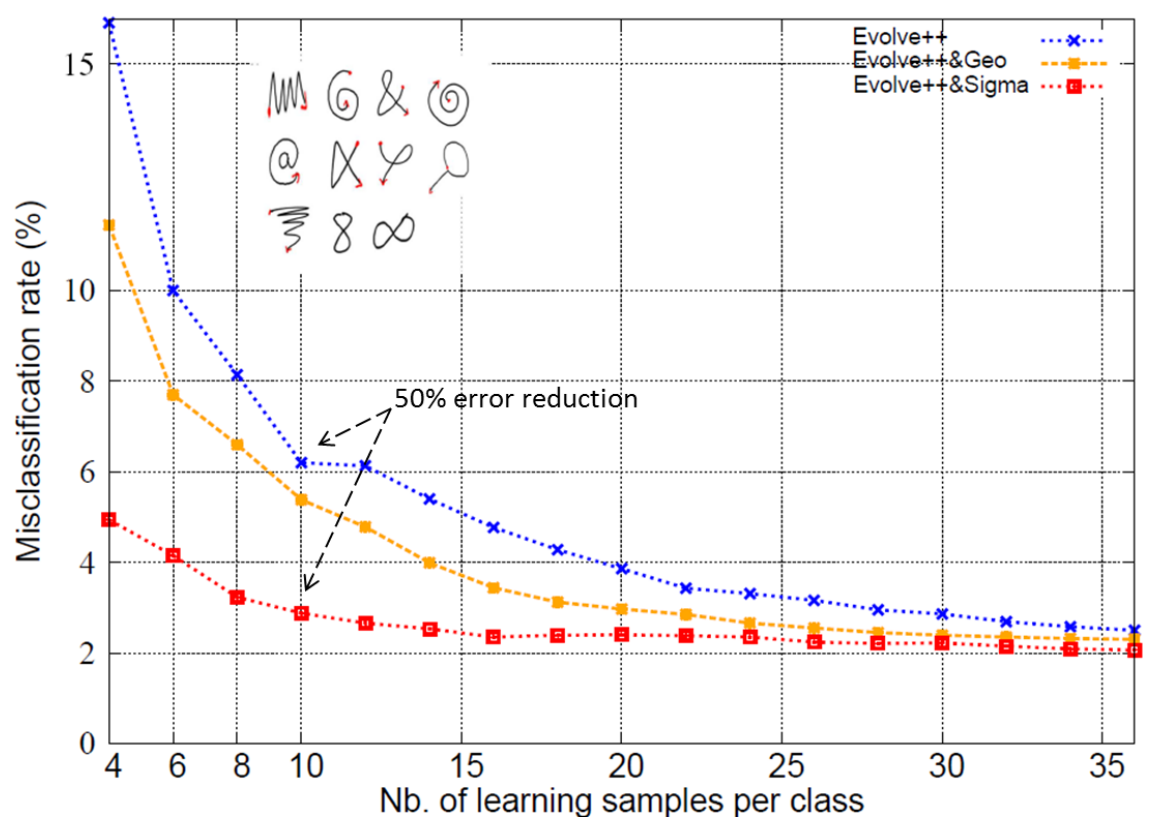

(a)

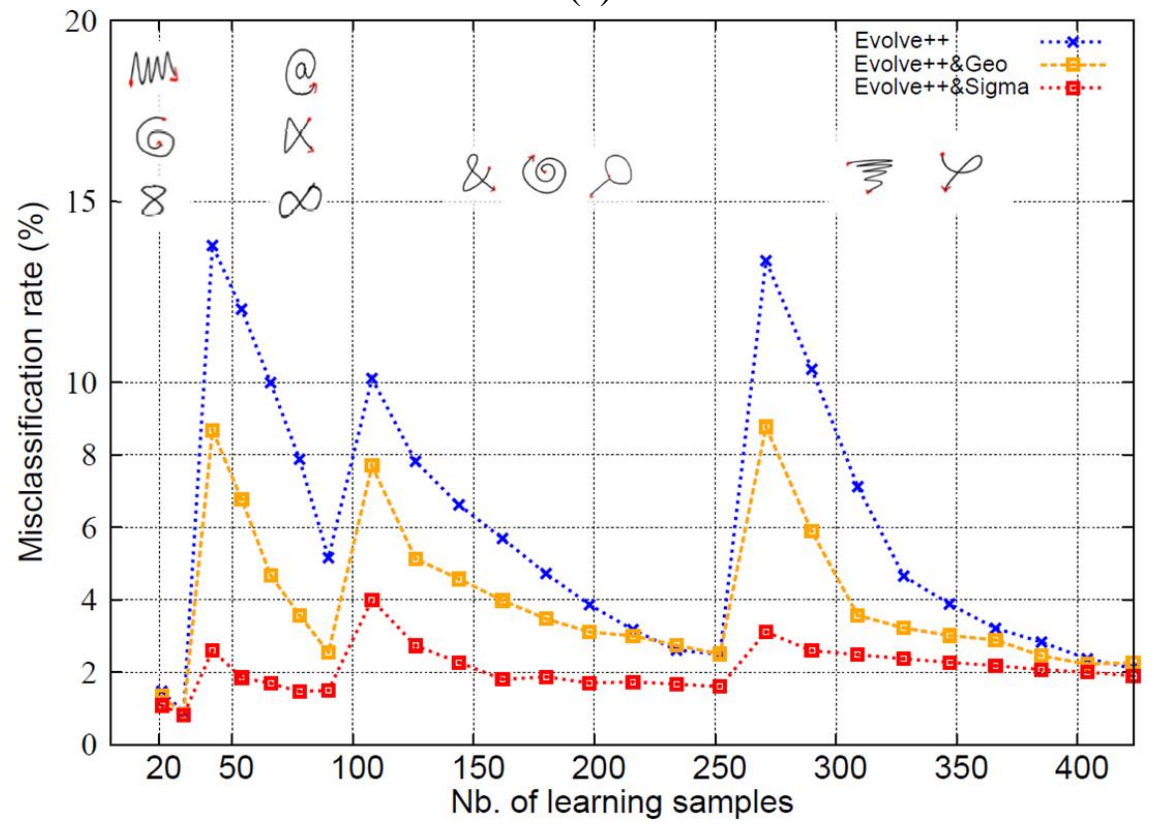

(b)

Fig. 9 (a) Performance improvement using synthetic data generation.

(b) Impact of synthetic samples when adding new gestures.

536 As it can be seen in Fig. 9.a, there is an important impact of the synthetic gestures at the 537 beginning of the incremental learning process. For example, the misclassification rate is 
538 reduced by $50 \%$ for 10 real samples per class when the training is enriched with synthetic

539 samples. It must also be noted from the same figure that distortions applied on the sigma-

540 lognormal parameters produces a more realistic variability in the synthetic gestures as

541 compared to direct geometrical distortions. Thanks to the realistic human-like distortions,

542 the synthetic samples present a significant ability to predict the appearance of future real

543 samples, which significantly accelerates the adaptation process. Fig. 9.b shows that using

544 synthetic samples, the classifier resists much better when introducing new classes. It is

545 able to rapidly re-estimate all its parameters and to improve the recognition performance

546 for the old and the new gestures. Again, the superiority of lognormal-based deformations

547 over the traditional geometrical ones is quite apparent.

548 These experimental results show that sigma-lognormal based synthetic samples play an 549 important role in improving the classification performance and accelerating the learning 550 process both when it starts from scratch and also when new gestures are introduced. One 551 interest of the present methodology is also that it does not depend on the way reference 552 gestures are defined and collected. For example, gestures that are recorded on hand held 553 mobile devices while the user is standing up, writing vertically, or while he is sitting in a 554 moving vehicle could also be used to train the system without really affecting the results.

\section{7. Conclusion}

557 In the last few years, great advances have been done on the problem of parameter 558 extraction of the Sigma-Lognormal model. New algorithms have been designed which 559 now allow an automatic representation of complex human motions such as those involved 560 in on-line signature and handwriting. The availability of these new systems and a better 
561 understanding of the variability of the sigma-lognormal parameters have paved the way

562 for the use of this model in the context of automatic generation of synthetic databases of

563 human movements. This paper has summarized the promising results of two different

564 investigations on that topic.

565 Needless to say that further research is still needed on the various topic addressed in this

566 paper, but as can already be seen, the use of the Sigma-Lognormal model for the

567 generation of human like movements offers very interesting perspectives for the field of

568 pattern recognition and the development of verification and recognition systems based on

569 human movements.

570

\section{Acknowledgements}

572 This work was supported by NSERC grant RGPIN-915 to Réjean Plamondon and a

573 NSERC PGS D scholarships to Christian O'Reilly. This work was also partially

574 supported by projects Contexts (S2009/TIC-1485) from CAM and Bio-Challenge

575 (TEC2009-11186) from Spanish MICINN. 


\section{REFERENCES}

578 Alimi, M. A., 2003. Beta neuro-fuzzy systems, in: W. Duch, D. Rutkowska (Eds.), TASK Quarterly J., 579 Special Issue on Neural Networks, vol. 7(1), pp. 23-41.

580 Almaksour, A. and Anquetil, E., 2011. Improving premise structure in evolving Takagi-Sugeno neuro581 fuzzy classifiers. Evolving Systems Journal, 2(1):25-33.

582 Bizzi, E., Hogan, N., Mussa-Ivaldi, F. A., Giszter, S., 1992. Does the nervous system use equilibrium-point 583 control to guide single and multiple joint movements?, Behav. Brain Sci. 15 603-613.

584 Bizzi, E., Dev, P., Morasso, P., and Polit, A., 1978 "Effect of Load Disturbances During Centrally Initiated 585 Movements," Journal of Neurophysiology, vol. 41, pp. 542-556.

586 Bullock and D., Grossberg, S., 1988. The VITE model: a neural command circuit for generating arm and 587 articulator trajectories, in: J.A.S. Kelso, A.J. Mandell, M.F. Shlesinger (Eds.), Dynamic Patterns in 588 Complex Systems, World Scientific Publishers, Singapore, pp. 305-326.

589 Cappelli, R., 2003. 'Synthetic Fingerprint Generation,' Handbook of Fingerprint Recognition, Springer, pp. $590 \quad 203-231$.

591 Cappelli, R., Maio, D., Maltoni, D., Wayman, J. L., and Jain, A. K., 2006. Performance evaluation of 592 fingerprint verification systems, IEEE Trans. on Pattern Analysis and Machine Intelligence 28, pp. 3-18.

593 Denier Van Der Gon J.J. and Thuring J.P.H., 1965. The guiding of human movements, Kybernetik, $594 \quad 14,145-148$

595 Djeziri, S., Guerfali, W., Plamondon, R. and Robert, J. M. 2002. Learning handwriting with pen-based 596 systems: computational issues. Pattern Recognition, Special Issue, 35(5), 1049-1057.

597 Djioua, M., Plamondon, R., 2007. Analysis and Synthesis of Handwriting Variability using the Sigma598 Lognormal Model. 13th Conference of the International Graphonomics Society. J. G. Philips, Rogers, D. \& 599 Ogeil, R.P. Melbourne, Victoria, Australia: 19-22.

600 Djioua, M., Plamondon, R., 2008. "A new methodology to improve myoelectric signal processing using 601 handwriting," presented at the International Conference on Frontiers in Handwriting Recognition, 602 Montreal, 2008, ICFHR'2008, pp. 112-117.

603 Djioua, M., Plamondon, R., 2009. "Studying the Variability of Handwriting Patterns using the Kinematic 604 Theory” Human Movement Science, vol. 28, No 5, pp. 588-601.

605 Dooijes, E.H., 1983. Analysis of handwriting movements , Acta Psychologica, 54, 99-114.

606 Edelman, S., Flash, T., 1987. A model of handwriting, Biol. Cybern. 57, 25-36.

607 Enderle, J. D. and Wolfe, J. W. 1987. Time-optimal control of saccadic eye movements. IEEE Transactions 608 on Biomedical Engineering, vol. 34, pp. 43-55.

609 Engelbrecht, S. E., 2001. Minimum principles in motor control, J. Math. Psychol. 45, 497-542.

610 Feldman, A. G. 1966. Functional tuning of the nervous system with control of movement or maintenance of 611 a steady posture. II. Controllable parameters of the muscle, Biophysics, vol. 11, pp. 565-578.

612 Feldman, A.G. and Latash, M.L., 2005. Testing hypotheses and the advancement of science: recent 613 attempts to falsify the equilibrium point hypothesis, Exp. Brain Res. 161 (1) 91-103. 
Feng, C., Woch, A., and Plamondon, R., 2002. "A Comparative Study of Two Velocity Profile Models for Rapid Stroke Analysis," Proceedings of the 16th International Conference on Pattern Recognition, Quebec, Canada, vol. 4, pp. 52-55.

Fierrez, J., Galbally, J., Ortega-Garcia, J., Freire, M. R., Alonso-Fernandez, F., Ramos, D., Toledano, D. T., Gonzalez-Rodriguez, J., Siguenza, J. A., Garrido-Salas, J., Anguiano, E. , Gonzalez-de-Rivera, G., Ribalda, R., Faundez-Zanuy, M., Ortega, J. A., Cardeñoso-Payo, V., Viloria, A., Vivaracho, C. E., Moro, Q. I., Igarza, J. J., Sanchez, J., Hernaez, I., Orrite-Uruñuela, C., Martinez-Contreras, F., and Gracia-Roche, J. J., 2010. BiosecurID: A Multimodal Biometric Database, Pattern Analysis and Applications, Vol. 13, n. 2, pp. 235-246.

Fierrez, J., Ortega-Garcia, J., Ramos, D., and Gonzalez-Rodriguez, J., 2007. HMM-based on-line signature verification: feature extraction and signature modeling, Pattern Recognition Letters, vol 8, pp. 2325-2334.

Flash, T. and Hogan, N., 1985. The coordination of Arm Movements: An experimentally confirmed mathematical model, The Journal of Neuroscience, vol. 5, pp. 1688-1703.

Flynn, P., 2008. Handbook of Biometrics, ch. 25. Springer, pp. 529-548.

Galbally, J., Fierrez, J., Martinez-Diaz, M., and Ortega-Garcia, J., 2009. "Synthetic Generation of Handwritten Signatures Based on Spectral Analysis", in Defense and Security Symposium, Biometric Technologies for Human Identification, BTHI, Proc. SPIE, Vol. 7306, pp. 730629.

Gangadhar, G., Joseph, D., Chakravarthy, V.S., 2007. An oscillatory neuromotor model of handwriting generation, Int. J. Doc. Anal. Recognition 10 (2), 69-84.

Grossberg, S. and Paine, R. W., 2000. A neural model of corticocerebellar interactions during attentive imitation and predictive learning of sequential handwriting movements, Neural Networks. 13 999-1046.

Harris, C.M. and Wolpert, D.M., 1998. Signal-dependent noise determines motor planning, Nature, 394,) 780-784.

Hermes, H. and LaSalle, J. P., 1969. Functional analysis and time optimal control. New York.

Hogan, N., 1984. "An organization principle for a class of voluntary movements," The Journal of Neurosciences, vol. 4, pp. 2745-2754.

Hollerbach, J.M., 1981. An oscillation theory of handwriting, Biol. Cybern. 39 (2) 139-156.

Kalveram, K.T., 1998. A neural oscillator model learning given trajectories, or how an allo-imitation algorithm can be implemented into a motor controller, in: J.P. Piek (Ed.), Motor Behavior and Human Skill: A Multidisciplinary Approach, Human Kinetics, pp. 127-140.

Kelso, S., 1995. Dynamic patterns: The self-organization of brain and behavior, MIT Press, Cambridge, MA. USA.

Kholmatov, A. and Yanikoglu, B., 2005. Identity authentication using improved online signature verification method, Pattern Recognition Letters, vol. 26, pp 2400-2408.

Leclerc, F., Plamondon, R., Lorette, G., 1992. Des gaussiennes pour la modélisation des signatures et la segmentation des tracés manuscrits, Traitement du Signal, vol. 9, no. 4, pp. 347-358.

Lin, Z. and Wan, L., 2007. Style-preserving English handwriting synthesis, Pattern Recognition, vol. 40, pp. 2097-2109.

Maarse, F.J., 1987. The Study of Handwriting Movement: Peripheral Models and Signal Processing Tehcniques, Swets \& Zertlinger, Lisse, The Netherlands. 

Springer, pp. 1185-1192.

657 Martinez-Diaz, M., Fierrez, J. and Hangai, S., 2009b. 'Signature matching,' Encyclopedia of biometrics, 658 Springer, pp. 1192-1196.

659 Mitoma, H., Uchida, S. and Sakoe, H., 2005. "Online character recognition based on elastic matching and 660 quadratic discrimination," in Proceedings of the Eighth International Conference on Document Analysis 661 and Recognition, ICDAR '05. Washington, DC, USA: IEEE Computer Society, pp. 36-40.

662 Morasso, P, Mussa Ivaldi, F.A. and Ruggiero, C., 1983. How a discontinuous mechanism can produce 663 continuous patterns in trajectory formation and handwriting, Acta Psychologica, 54, 83-98.

664 Mouchère, H., Bayoudh, S., Anquetil, E and Miclet, L., 2007. "Synthetic on-line handwriting generation by 665 distortions and analogy," in 13th Conference of the International Graphonomics Society (IGS2007), 666 November 2007, pp. 10-13.

667 Neilson, P.D. and Neilson, M.D., 2005. An overview of adaptive model theory: solving the problems of 668 redundancy, resources and nonlinear interactions in human movement control, J. Neural Eng. 2 (3) 279669312.

670 Neilson, P.D., 1993. The problem of redundancy in movement control: the adaptive model theory 671 approach, Psychol. Res. 55, 99-106.

672 Nelson, W.L., 1983. Physical principles for economies of skilled movements, Biol. Cybern. 46 135-147.

673 O'Reilly, C. and Plamondon, R., 2007. "A software assistant for the design and analysis of neuromuscular 674 tests," Proc. IEEE Biomedical Circuits and Systems Conference, Nov. 2007, pp. 107 - 110.

675

676 Signatures. The 11th International Conference on Frontiers in Handwriting Recognition, Montreal, Canada, CENPARMI. pp. 216-222.

O'Reilly, C. and Plamondon, R., 2009. Development of a Sigma-Lognormal representation for on-line signatures. Pattern Recognition 42(12): 3324-3337.

O'Reilly, C. and Plamondon, R., 2010. Prototype-based methodology for the statistical analysis of local features in stereotypical handwriting tasks. International Conference on Pattern Recognition, Istanbul, Turkey, pp. 18641867.

O'Reilly, C. and Plamondon, R., 2011. Impact of the principal stroke risk factors on human movements. Human Movement Science 30(4): 792-806.

O'Reilly, C. and Plamondon, R. 2012. Design of a Neuromuscular Disorders Diagnostic System Using Human Movement Analysis. Paper accepted for presentation at the The 11th International Conference on Information Sciences, Signal Processing and their Applications, Montreal, Canada, July 3-5 2012.

O'Reilly, C., 2012. Développement d'outils d'analyse de la motricité fine pour l'investigation de troubles neuromusculaires: Théorie, prototype et mise en application dans le contexte des accidents vasculaires cérébraux. Génie Électrique. Montréal, École Polytechnique. Ph.D, in preparation.

Ortega-Garcia, J., Fierrez-Aguilar, J., Simon, D., Gonzalez, J., Faundez, M., Espinosa, V., Satue, A., Hernaez, I., Igarza, J. J., Vivaracho, C., Escudero, D. and Moro, Q. I., 2003. "MCYT baseline corpus: A bimodal biometric database", IEE Proceedings Vision, Image and Signal Processing, Special Issue on 701 Biometrics on the Internet, Vol. 150, n. 6, pp. 395-401 
Plamondon, R., 1994. The Design of An On-Line Signature Verification System: From Theory to Practice. International Journal of Pattern Recognition and Artificial Intelligence, Special Issue on Signature Verification, 8 (3): 795-811.

Plamondon, R., Li, X. and Djioua, M., 2007. "Extraction of delta-lognormal parameters from handwriting strokes," Journal of Frontiers of Computer Science in China, vol. 1(1), pp. 106-113.

Plamondon, R. and Lamarche, F., 1986. "Modelization of Handwriting: A System approach", in Graphonomics: Contemporary Research in Handwriting, H.S.R. Kao, G.P. van Galen and R. Hoosain, Eds. Elsevier, Amsterdam., pp 169-183.

Plamondon, R. and Djioua, M., 2006. "A multi-level representation paradigm for handwriting stroke generation" Human Movement Science, vol. 25, pp. 586-607.

Plamondon, R., Feng, C. and Woch, A., 2003. A kinematic theory of rapid human movement. Part IV: a formal mathematical proof and new insights, Biological Cybernetics, vol. 89, pp. 126-138.

Plamondon, R. and Guerfali, W., 1998. The generation of handwriting with delta-lognormal synergies, Biological Cybernetics, vol. 78, pp. 119-132.

Plamondon, R. and Alimi, A., 1997. Speed/Accuracy tradeoffs in target-directed movements, Behavioral and Brain Sciences, vol. 20, pp. 279-349.

Plamondon, R. and Privitera, C.M., 1995. A Neural Model for Generating and Learning a Rapid Movement Sequence, Biological Cybernetics, vol. 74, No 2, p. 117-130.

Plamondon, R., 1995a. A kinematic theory of rapid human movements. I. Movement representation and generation, Biol. Cybern. 72 (4) 295-307.

Plamondon, R., 1995b. A kinematic theory of rapid human movements. II. Movement time and control, Biol. Cybern. 72 (4) 309-320.

Pudil, P, Novovicova, J. and Kittler, J., 1994. Flotating search methods in feature selection, Pattern Recognition Letters, vol 15, pp. 1119-1125.

Schmidt, R.A., Lee, T.D, 1999. Motor Control and Learning: A behavioral Emphasis, 3rd Ed, Human Kinetics, Champaign Illinois, 493p.

Shah, S. and Ross, A., 2006. Generating synthetic irises by feature agglomeration, in: Proc. IEEE Int. Conf. on Image Processing (ICIP), pp. 317-320.

Schomaker, L.R.B., 1991. Simulation and recognition of handwriting movement: a vertical approach to modeling human motor behavior, PhD Thesis, Nijmegen University, Netherlands.

Tanaka, H., Krakauer, J.W. and Qian, N., 2006. An optimization principle for determining movement duration, J. Neurophysiol. 953875-3886.

Thomassen, A.J.W.M., Keuss, P.J.G. and van Galen, G.P., 1983. "Motor aspects of handwriting”, Acta Psychologica, vol 54.

Uno, Y., Kawato, M. and Suzuki, R., 1989. Formation, Control of optimal trajectory in human multijoint arm movement, Biol. Cybern. 61 89-101.

van Galen, G.P. and Teulings, H.L.T., 1983. The independent monitoring of form and scale factors in Handwriting, in Motor Aspects of Handwriting, North Holland, Amsterdam, The Netherlands. 
743 Wada, Y. and Kawato, M., 1995. A theory for cursive handwriting based on the minimization principle, 744 Biol. Cybern. 73 (1) 3-13.

745 Wang, J., Wu, C., Xu, Y.-Q., and Shum, H.-Y.,2005. "Combining shape and physical models for online 746 cursive handwriting synthesis," International Journal on Document Analysis and Recognition, vol. 7, pp. $747 \quad 219-227$.

748 Wise, M.E., 1966. The geometry of lognormal and related distributions and an application to tracer-dilution curves, Statistica Neerlandica, vol. 20, no.1.

Woch, A., 2006. "Étude des primitives bidirectionnelles du mouvement dans le cadre de la théorie cinématique: confirmation expérimentale du modèle delta-lognormal," in Département de Génie Électrique. Montréal École Polytechnique.

754 Zanone, P-G., Athènes, S., Sallagoity, I. and Albaret, J-M., 2005. Switching Among Graphic Patterns is 755 Governed by Coordination Dynamics of Handwriting, Proc. $12^{\text {th }}$ Biennial Conf. of the Int. Graphonomics 756 Society, Salerno, Italy, pp 255-260.

757 Zuo, J., Schmid, N. A. and Chen, X., 2007. On generation and analysis of synthetic iris images, IEEE 758 Trans. on Information Forensics and Security, vol 2, pp. 77-90. 